\title{
Distribución espacio - temporal de Ephemeroptera (Arthropoda: Insecta) en ríos de Yungas de Jujuy (Argentina)
}

\section{Spatio - temporal distribution of Ephemeroptera (Arthropoda: Insecta) in Yungas rivers of Jujuy (Argentina)}

\author{
Marcela Alejandra De Paul ${ }^{1}$, Lilia Estela Neder de Román², Carmen Inés Viturro³, \\ Juan Pablo Villafañe ${ }^{1}$
}

\begin{abstract}
${ }^{1}$ Centro de Estudios Interdisciplinarios de Calidad de Aguas de Ambientes de Altura, Facultad de Ciencias Agrarias, Universidad Nacional de Jujuy. Alberdi 47. 4600 San Salvador de Jujuy. Argentina. ${ }^{2}$ Sección Entomología. Instituto de Biología de la Altura. Universidad Nacional de Jujuy. Av. Bolivia 1661. 4600. San Salvador de Jujuy. Argentina.

3Laboratorio PRONOA, Facultad de Ingeniería, CIITeD-CONICET, Universidad Nacional de Jujuy, Ítalo Palanca 10, San Salvador de Jujuy, Argentina.
\end{abstract}

E-mail: marceladepaul@hotmail.com

RESUMEN. Los ríos que conforman la cuenca del río Reyes pertenecen a la ecorregión de las Yungas de Jujuy (Argentina). Con el objetivo de identificar la representatividad, riqueza y variación espacio temporal del orden Ephemeroptera en la cuenca se consideraron 10 sitios en el perfil longitudinal (1380- 2200 m s.n.m.) de los ríos Reyes, Lampazar, Quesera, Chinchuli, Guerrero, en dos años (2010-2011) y dos etapas del ciclo hidrológico: aguas altas (marzo -abril: otoño) y aguas bajas (octubre - noviembre: primavera). Se registró una alta representatividad del orden (76,3\%) y familias (Baetidae: 72,2\%; Leptohyphidae: 3,1\%; Leptophlebiidae 1,4\% y Caenidae: 0,004\%) sobre el total del bentos. Se analizó la variabilidad espacio - temporal de la abundancia de ocho especies. Se registró un cambio en la representatividad estacional del orden ( $87 \%$ en otoño y $71 \%$ en primavera) y de las especies. Se registraron in situ, variables fisicoquímicas del agua -temperatura, $\mathrm{pH}$, conductividad y oxígeno disuelto- que permitieron explicar el $70 \%$ de la variabilidad espacio - temporal de los sitios mediante análisis de componentes principales. El análisis de correlación canónica mostró que la abundancia de las especies se relaciona en forma significativa con los parámetros fisicoquímicos registrados $\left(R^{2}=0,76 ; p=0,001\right)$. Se comprobó la importancia de los Ephemeroptera y el desempeño de cada familia y especie, como indicadoras de condiciones particulares del agua, así como también su variación espacio - temporal característica de las Yungas de Jujuy y el rango de tolerancia definido por los valores fisicoquímicos en que fueron registradas.

Palabras clave: Distribución, Ephemeroptera, estacionalidad, parámetros fisicoquímicos, ríos de montaña, Yungas.

ABSTRACT. The rivers of Reyes basin belong to the Yungas ecoregion (Jujuy-Argentina). To identify the representativeness, richness and spatio-temporal variation of the Ephemeroptera order in the basin, 10 sites were investigated in the longitudinal profile (1380-2200 m a.s.l.) of the rivers Reyes, Lampazar, Quesera, Chinchuli, Guerrero, in two years (2010-2011) and two stages of the hydrological cycle: high waters (March-April: autumn) and low waters (October-November: spring). A high representativeness of the order (76.3\%) and families (Baetidae: 72.2\%; Leptohyphidae: 3.1\%; Leptophlebiidae $1.4 \%$ and Caenidae: $0.004 \%$ ) was observed in relation to total benthos. The spatiotemporal variability of the abundance of eight species was analysed. A change in the seasonal representativeness of the order ( $87 \%$ in autumn and $71 \%$ in spring) and species was detected. In situ, physical chemical variables of the water were recorded - temperature, $\mathrm{pH}$, conductivity, dissolved oxygen - which allowed explaining $70 \%$ of the spatio - temporal variability of the sites, by means 
of principal component analysis. The canonical correlation analysis showed that the abundance of the species is significantly related to the physicochemical parameters recorded $\left(R^{2}=0.76 ; p=0.001\right)$. The importance of the Ephemeroptera and the role of each family and species as indicators of particular water conditions were confirmed. Also, the spatio-temporal variation characteristic of the Yungas of Jujuy was recognized, establishing the tolerance range of the species defined by the recorded physicochemical parameters.

Keywords: Distribution, Ephemeroptera, mountain rivers, physical and chemical parameters, seasonality, Yungas.

\section{INTRODUCCIÓN}

Los Ephemeroptera presentan una amplia distribución mundial a excepción de la Antártida, colonizando los ambientes de agua dulce y, a veces, salobre (Sartori \& Brittain, 2015). En los sistemas de agua dulce, los Ephemeroptera desempeñan funciones esenciales en las redes alimenticias al procesar material orgánico y servir como fuente primaria de alimentos para otros animales (Wallace \& Webster, 1996). La temperatura del agua es el factor de mayor peso para regular la tasa de desarrollo y crecimiento (Salles et al., 2018). Las familias, Baetidae, Caenidae y Leptophlebiidae son cosmopolitas, mientras que Leptohyphidae presenta una distribución americana (Sartori \& Brittain, 2015). Los Baetidae incluyen más de 650 especies (Cruz et al., 2014), colonizando sus ninfas diferentes hábitats de agua dulce con gran número de individuos (Domínguez y Fernández, 2009).

El interés por conocer la calidad ecológica del agua y su evolución en el tiempo ha generado la construcción de numerosos índices basados en valoraciones cuantificables de la tolerancia de los macroinvertebrados bentónicos, siendo el orden Ephemeroptera, uno de los más valorados. Sin embargo, las familias del orden presentan diferentes grados de tolerancia a los cambios (Domínguez y Fernández, 1998; Dos Santos et al., 2011; Mesa, 2011; Studholme et al., 2014). El presente estudio tiene como objetivo analizar la representatividad, riqueza y variación espacio temporal del orden Ephemeroptera en la cuenca del Reyes, considerada como referencia para las Yungas occidentales de Jujuy, en dos estaciones del año marcadamente diferentes desde el punto de vista hidrológico (aguas altas - aguas bajas).
Para identificar su capacidad como indicadores de condiciones particulares del agua, se efectúa la correlación entre los registros fisicoquímicos y las abundancias de las especies.

\section{MATERIALES Y MÉTODOS Área de estudio}

La cuenca del río Reyes se encuentra en la Cordillera Oriental de Jujuy, departamento General Belgrano entre los $24^{\circ} 05^{\prime} 21^{\prime \prime} \mathrm{S}$, $24^{\circ} 18^{\prime} 02^{\prime \prime} \mathrm{S}$ y los $65^{\circ} 43^{\prime} 22^{\prime \prime} \mathrm{O}, 6^{\circ} 22^{\prime} 47^{\prime \prime} \mathrm{O}$. El área aproximada es de $450 \mathrm{~km}^{2}$, sus cabeceras se ubican en la Serranía del Chañi, a los 5517 msnm, desembocando a los 1350 msnm en el río Grande. En la cuenca discurren cinco ríos: Reyes, Quesera, Lampazar, Guerrero y Chinchuli. En la cuenca del río Reyes se distinguen dos subcuencas 1) Reyes: con una superficie estimada de $301 \mathrm{~km}^{2}$, pendiente del 26,7 \%, 713 cursos de agua de orden 1 (clasificación de Sthraler) y un coeficiente de torrencialidad de 2,3. 2) Guerrero: superficie aproximada $148,6 \mathrm{~km}^{2}$, pendiente del 13\%, 184 cursos de orden 1 y coeficiente de torrencialidad de 1,3. Los ríos corren de oeste a este y presentan características torrenciales con gran capacidad de arrastre y erosión. Poseen lecho pedregoso con amplias playas de inundación (50-100 m), presencia de variados microclimas en función de la altitud, exposición solar y pendiente. El régimen hidrológico de los ríos que la componen es predominantemente pluvial (noviembre a abril), experimentando amplias variaciones de caudal a lo largo del año. La zona en estudio se caracteriza por veranos cálidos y húmedos y por inviernos secos, oscilando las temperaturas máximas 
y mínimas entre los $30^{\circ} \mathrm{C}$ y $0^{\circ} \mathrm{C}$ (Braun Wilke, 2001). Fitogeográficamente las nacientes de los ríos pertenecen a la provincia Altoandina mientras que la parte baja y media de la cuenca corresponde a la provincia de las Yungas (Cabrera, 1976).

\section{Muestreo y procesamiento de muestras}

Se fijaron diez estaciones de muestreo considerando cada uno de los ríos que conforman la cuenca (ríos Reyes, Quesera, Lampazar, Guerrero, Chinchuli) (Figura 1, Tabla 1) que fueron visitados dos veces al año: otoño (aguas altas: fin de período de lluvias) y primavera (aguas bajas: caudales mínimos al malla, durante diez minutos mediante técnica de pateo. Se colectaron también imagos con el fin de facilitar la identificación de las ninfas. El material fue conservado en frascos plásticos en alcohol al 96\%. En el laboratorio, las muestras fueron separadas, los ejemplares cuantificados e identificados al menor nivel taxonómico, con la ayuda de una lupa estereoscópica Leica EZ4 y Nikon SMZ 800, utilizando oculares 10x y 20x. Para su identificación se emplearon claves de macroinvertebrados sudamericanos (Domínguez, 1984; Domínguez y Fernández, 2009; Hamada et al., 2018; Molineri, 2010; Molineri \& Malzacher, 2007; Nieto, 2003, 2004; Pescador \& Peters, 1986). Los ejemplares se encuentran depositados en el CEICAAL -UNJU

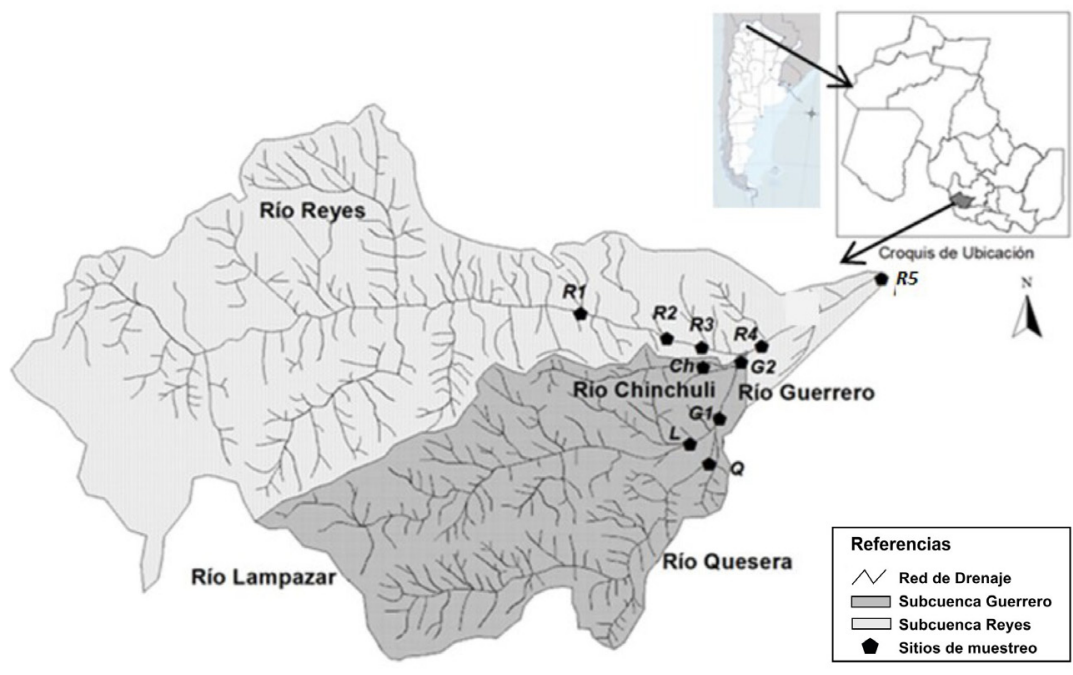

Figura 1. Ubicación de los sitios muestreados. Cuenca del río Reyes, Jujuy, Argentina.

Figure 1. Location of the sampled sites. Reyes basin, Jujuy, Argentine.

final de la época seca) y durante dos años (20102011). Para la subcuenca Reyes se seleccionaron cinco sitios, desde mayor a menor altitud: 2200 (máxima altitud accesible) - $1380 \mathrm{msnm}$ ): R1, R2, R3, R4, R5. Para la subcuenca Guerrero los sitios de muestreo fueron cinco: Q, L, G1, G2, Ch (1621- 1509 m s.n.m.).

Para obtener datos cuantitativos de los organismos en cada punto de muestreo se utilizó una red Surber (tres réplicas por sitio) con $250 \mu \mathrm{m}$ de abertura de malla. Para incorporar especies presentes en otros hábitats del río se utilizó una red D con la misma abertura de
(Centro de Estudios Interdisciplinarios de Calidad de Agua de Ambientes de Altura, Universidad Nacional de Jujuy, Jujuy, Argentina). De manera simultánea al muestreo de macroinvertebrados, se midieron parámetros físico y químicos del agua mediante sonda multiparamétrica Cole Parmer PCD650: $\mathrm{pH}$, temperatura del agua $\left({ }^{\circ} \mathrm{C}\right)$, conductividad $(\mu \mathrm{S} /$ $\mathrm{cm})$, oxígeno disuelto ( $\mathrm{mg} / \mathrm{L})$.

\section{Tratamiento de datos}

Los sitios se codificaron con las siglas que 
De Paul et al.

Tabla 1. Sitios de muestreo y parámetros ecológicos y físicoquímicos registrados.

Table 1. Sampling sities an ecological recorded and physicochemical parameters.

\begin{tabular}{|c|c|c|c|c|}
\hline $\begin{array}{l}\text { Sub- } \\
\text { cuenca }\end{array}$ & Río/ sitio & $\begin{array}{c}\text { Coordenadas } \\
\text { Geográficas }\end{array}$ & $\begin{array}{c}\text { Altitud } \\
\text { (m s.n.m.) }\end{array}$ & Características \\
\hline & Reyes (R1) & $\begin{array}{l}24^{\circ} 9^{\prime} 40^{\prime \prime} \mathrm{S} \\
65^{\circ} 32^{\prime} 26.4^{\prime \prime} \mathrm{W}\end{array}$ & 2190 & $\begin{array}{l}\text { Toma de agua para central termoeléctrica: caudal regulado. Sustrato: } \\
\text { predominio de arenas y canto rodado. Vegetación riparia dispersa: } \\
\text { pastizales. }\end{array}$ \\
\hline & Reyes (R2) & $\begin{array}{l}24^{\circ} 10^{\prime \prime} 5.2^{\prime \prime} \mathrm{S} \\
65^{\circ} 29^{\prime \prime} 34.8^{\prime \prime} \mathrm{W}\end{array}$ & 1784 & $\begin{array}{l}\text { Recibe arroyo Puerta de Hacha. Sustrato: predominio de canto rodado. } \\
\text { Vegetación riparia dispersa y alejada de los márgenes: alisos. }\end{array}$ \\
\hline & Reyes (R3) & $\begin{array}{l}24^{\circ} 10^{\prime} 40.1^{\prime \prime} \mathrm{S} \\
65^{\circ} 27^{\prime} 37.9^{\prime \prime} \mathrm{W}\end{array}$ & 1609 & $\begin{array}{l}\text { Aguas abajo de establecimiento recreativo: recibe efluentes cloacales. } \\
\text { Asentamientos urbanos dispersos. Predominio de canto rodado. } \\
\text { Vegetación riparia: alisos. }\end{array}$ \\
\hline 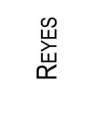 & Reyes (R4) & $\begin{array}{l}24^{\circ} 10^{\prime} 59.7^{\prime \prime} \mathrm{S} \\
65^{\circ} 25^{\prime} 43^{\prime \prime} \mathrm{W}\end{array}$ & 1491 & $\begin{array}{l}\text { Aguas abajo de la confluencia con el río Guerrero. Extracción de } \\
\text { caudal para planta potabilizadora. Extracción de áridos. El cauce } \\
\text { principal se divide en brazos menores. Vegetación riparia modificada } \\
\text { por el hombre. }\end{array}$ \\
\hline
\end{tabular}

Reyes (R5) $\quad 24^{\circ} 9 " 34.4^{\prime \prime} \mathrm{S}$
$65^{\circ} 22 " 58.5^{\prime \prime} \mathrm{W}$

Punto de drenaje de la cuenca, antes de desembocar en el río Grande. Aguas abajo de pueblo de San Pablo de Reyes: efluentes domésticos, actividades antrópicas evidentes: basura dispersa. Alteración de la morfología del cauce y del lecho por la construcción de puente. Sustrato: canto rodado. Vegetación riparia degradada.

\begin{tabular}{|c|c|}
\hline Quesera (Q) & $\begin{array}{l}20^{\circ} 12 " 48.5^{\prime \prime} \mathrm{S} \\
65^{\circ} 26^{\prime} 40.7^{\prime \prime} \mathrm{W}\end{array}$ \\
\hline $\begin{array}{l}\text { Lampazar } \\
\text { (L) }\end{array}$ & $\begin{array}{l}24^{\circ} 12 " 48.8^{\prime \prime} \mathrm{S} \\
65^{\circ} 26^{\prime \prime} 45.2^{\prime \prime} \mathrm{V}\end{array}$ \\
\hline Guerrero (G1) & $\begin{array}{l}24^{\circ} 12^{\prime} 4,8^{\prime \prime} \mathrm{S} \\
65^{\circ} 26^{\prime} 20,5^{\prime \prime} \mathrm{W}\end{array}$ \\
\hline $\begin{array}{l}\text { Guerrero } \\
\text { (G2) }\end{array}$ & $\begin{array}{l}24^{\circ} 11^{\prime} 34,3^{\prime \prime} \\
65^{\circ} 26^{\prime \prime} 13,4^{\prime \prime} !\end{array}$ \\
\hline $\begin{array}{l}\text { Chinchuli } \\
\text { (CH) }\end{array}$ & $\begin{array}{l}24^{\circ} 11^{\prime} 38,2^{\prime \prime} \mathrm{S} \\
65^{\circ} 26^{\prime} 42,9^{\prime \prime} \mathrm{W}\end{array}$ \\
\hline
\end{tabular}

Naciente del río Guerrero. Sustrato: canto rodado. Vegetación riparia: alisos. Sitio con acceso restringido a personas.

Naciente del río Guerrero. Sitio con acceso restringido a personas. Sustrato: canto rodado. Vegetación riparia: alisos.

Nace por la unión de los ríos Quesera y Lampazar. Extracción de caudal para suministro de planta potabilizadora. Sitio con acceso restringido a personas. Sustrato: canto rodado. Vegetación riparia: alisos.

Aguas abajo de la confluencia con el río Chinchuli. Presencia de actividad urbana, basura. Caudal bajo todo el año por extracción continua para abastecimiento de planta potabilizadora. Sustrato: canto rodado. Vegetación riparia degradada.

Actividades urbanas dispersas y ganadería. Sustrato: canto rodado vegetación riparia degradada. relacionan su nombre, altitud (Reyes y Guerrero el 1 indica el de mayor altitud); la estación del año (O: otoño; P: primavera); el año de muestreo: 2010, 2011. Los datos obtenidos de las muestras Surber fueron promediados y expresados como abundancia relativa. Para identificar la variabilidad espacio - temporal de los sitios respecto de los registros de parámetros fisicoquímicos del agua se efectuó un análisis de componentes principales (programa Infostat, Di Rienzo et al., 2008). Previo al análisis, los registros fueron estandarizados $\left(\log _{10}\right)$ a excepción del pH. Para evaluar el papel de los Ephemeroptera como indicadores de calidad de agua se analizó la relación entre los registros de parámetros físicoquímicos vs. abundancia absoluta de cada especie mediante correlación canónica (programa estadístico Infostat, D Rienzo et al., 2008).

\section{RESULTADOS}

Se identificaron 14 especies pertenecientes a cuatro familias: Americabaetis alphus Lugo-Ortiz and McCafferty, 1996; Andesiops peruvianus Ulmer 1920; Baetodes cochunaensis Nieto 2004; Baetodes copiosus Nieto 2004; Baetodes huaico Nieto 2004; Camelobaetidius penai Traver and Edmunds 1968; Nanomis galera Lugo-Ortiz and McCafferty 1999 (Baetidae); Caenis Iudicra Navás 1920 (Caenidae); Haplohyphes baritu Domínguez 1984; Leptohyphes eximius Eaton 1882 (Leptohyphidae); Meridialaris tintinnabula 
Pescador and Peters 1987; Thraulodes cochunaensis Domínguez 1987; Thraulodes consortis Domínguez 1987; Thraulodes liminaris Domínguez 1987 (Leptophlebiidae) (Tabla 2 y Tabla 3).

En la cuenca del río Reyes, del total de macroinvertebrados recolectados $(n=85930)$, la clase Insecta representó el 99,4\% durante los otoños y el 95,1\% en primavera. Los efemerópteros $(n=65552)$ representaron el $76,3 \%$ de Insecta, variando su representatividad desde $86,8 \%$ en los otoños a $71,4 \%$ en primavera. Respecto a la representatividad de las familias y riqueza de especies, se destaca que Baetidae presentó la mayor riqueza de géneros y abundancia relativa $(72,2 \%$ de la comunidad completa de macroinvertebrados bentónicos). Con valores decrecientes de representatividad, Leptohyphidae obtuvo el 3,14\% con dos especies, Leptophlebiidae representó el $1,44 \%$ con cuatro especies y Caenidae el $0,004 \%$ con una sola especie. En la cuenca del Reyes la familia Baetidae registró siete especies, siendo el género Baetodes el mejor representado en la cuenca con tres especies: B. cochunaensis, B.copiosus, B. huaico. El género evidenció una amplia tolerancia a las variaciones en las condiciones físicoquímicas del agua y al flujo de las aguas. Alcanzó la mayor representatividad entre los 1400-1700 msnm. Con este estudio se amplía la distribución altitudinal para B. cochunaensis hasta los 2200

Tabla 2. Abundancia relativa (\%) de las especies de Ephemeroptera en las subcuencas Reyes y Guerrero en 2010 y 2011. (Otoño).

Table 2. Relative abundance (\%) of the Ephemeroptera species in the Reyes and Guerrero subbasins in 2010 and 2011. (Autumn).

\begin{tabular}{|c|c|c|c|c|c|c|c|c|c|c|}
\hline \multirow[b]{2}{*}{ OTOÑO } & \multicolumn{2}{|r|}{ R1 } & \multicolumn{2}{|c|}{ R2 } & \multicolumn{2}{|r|}{ R3 } & \multicolumn{2}{|r|}{ R4 } & \multicolumn{2}{|c|}{ R5 } \\
\hline & 2010 & $0 \quad 2011$ & 2010 & 2011 & 2010 & 2011 & $201 C$ & 2011 & 2010 & 2011 \\
\hline Americabaetis alphus & 0,0 & 0,0 & 0,0 & 0,0 & 0,2 & 0,0 & 0,0 & 0,0 & 0,1 & 0,0 \\
\hline Andesiops peruvianus & 27,7 & 18,9 & 0,6 & 23,1 & 10,7 & 1,0 & 0,8 & 1,4 & 4,0 & 0,2 \\
\hline Baetodes spp. & 17,3 & 6,8 & 91,6 & 51,6 & 11,8 & 68,1 & 84,1 & 79,9 & 86,7 & 93,1 \\
\hline Camelobaetiudius penai & 1,3 & 2,7 & 1,9 & 1,6 & 1,1 & 3,9 & 2,6 & 3,8 & 2,2 & 0,2 \\
\hline Nanomis galera & 0,0 & 0,0 & 0,0 & 0,0 & 2,2 & 0,0 & 0,2 & 1,0 & 0,2 & 0,1 \\
\hline Meridialaris tintinnabula & 2,2 & 1,4 & 0,1 & 0,7 & 0,1 & 0,0 & 0,1 & 0,6 & 0,3 & 0,9 \\
\hline Thraulodes cochunaensis & 0,9 & 0,0 & 0,2 & 0,0 & 0,0 & 0,0 & 0,0 & 0,0 & 0,0 & 0,0 \\
\hline Thraulodes consortis & 0,0 & 0,0 & 0,0 & 0,0 & 0,2 & 0,0 & 0,1 & 0,0 & 0,2 & 0,0 \\
\hline Thraulodes liminaris & 0,0 & 0,0 & 0,0 & 0,0 & 0,0 & 0,0 & 0,0 & 0,0 & 0,0 & 0,0 \\
\hline Haplohyphes baritu & 0,0 & 0,0 & 0,0 & 0,0 & 0,0 & 0,0 & 0,0 & 0,0 & 0,0 & 0,0 \\
\hline Leptohyphes eximius & 0,0 & 0,0 & 0,0 & 0,0 & 1,2 & 0,8 & 0,2 & 0,1 & 0,0 & 0,0 \\
\hline \multirow[t]{2}{*}{ Caenis ludrica } & 0,0 & 0,0 & 0,0 & 0,0 & 0,0 & 0,0 & 0,0 & 0,0 & 0,0 & 0,0 \\
\hline & \multicolumn{2}{|c|}{$Q$} & \multicolumn{2}{|c|}{ L } & \multicolumn{2}{|c|}{ G1 } & \multicolumn{2}{|c|}{ G2 } & \multicolumn{2}{|c|}{$\mathrm{Ch}$} \\
\hline OTOÑO & 2010 & 2011 & 2010 & 2011 & 2010 & 2011 & 2010 & 2011 & 2010 & 2011 \\
\hline Americabetis alphus & 0,0 & 0,0 & 0,0 & 0,0 & 0,2 & 0,0 & 0,0 & 0,0 & 0,1 & 0,0 \\
\hline Andesiops peruvianus & 1,2 & 1,7 & 2,0 & 0,3 & 2,4 & 1,1 & 1,5 & 0,2 & 1,3 & 0,0 \\
\hline Baetodes spp. & 71,1 & 71,9 & 72,0 & 75,1 & 60,7 & 74,7 & 72,6 & 86,6 & 67,4 & 91,1 \\
\hline Camelobaetidius penai & 11,3 & 6,2 & 7,5 & 6,6 & 17,9 & 5,8 & 12,6 & 4,4 & 18,4 & 3,4 \\
\hline Nanomis galera & 0,0 & 0,0 & 0,0 & 0,0 & 0,0 & 0,0 & 0,2 & 0,1 & 0,6 & 0,0 \\
\hline Meridialaris tintinnabula & 3,8 & 9,3 & 2,9 & 8,4 & 5,0 & 11,1 & 3,5 & 3,7 & 0,0 & 0,2 \\
\hline Thraulodes cochunaensis & 0,2 & 0,0 & 0,0 & 0,0 & 0,1 & 0,0 & 0,1 & 0,0 & 0,0 & 0,0 \\
\hline Thraulodes consortis & 0,0 & 0,0 & 0,0 & 0,0 & 0,1 & 0,0 & 0,1 & 0,0 & 0,0 & 0,0 \\
\hline Thraulodes liminaris & 0,1 & 0,0 & 0,0 & 0,0 & 0,0 & 0,0 & 0,0 & 0,0 & 0,0 & 0,0 \\
\hline Haplohyphes baritu & 0,0 & 0,0 & 0,0 & 0,0 & 0,0 & 0,0 & 0,0 & 0,0 & 0,0 & 0,0 \\
\hline Leptohyphes eximius & 0,0 & 0,0 & 0,3 & 0,2 & 0,0 & 0,1 & 0,7 & 0,0 & 0,1 & 0,1 \\
\hline Caenis ludrica & 0,0 & 0,0 & 0,0 & 0,0 & 0,0 & 0,0 & 0,0 & 0,0 & 0,0 & 0,0 \\
\hline
\end{tabular}


Tabla 3. Abundancia relativa (\%) de las especies de Ephemeroptera en las subcuencas Reyes y Guerrero en 2010 y 2011. (Primavera).

Table 3. Relative abundance (\%) of the Ephemeroptera species at the Reyes and Guerrero subbasins in 2010 and 2011. (Spring).

\begin{tabular}{|c|c|c|c|c|c|c|c|c|c|c|c|}
\hline \multirow[b]{2}{*}{ PRIMAVERA } & \multicolumn{2}{|c|}{ R1 } & \multicolumn{2}{|c|}{ R2 } & \multicolumn{2}{|r|}{ R3 } & \multicolumn{3}{|c|}{ R4 } & \multicolumn{2}{|r|}{ R5 } \\
\hline & 2010 & 2011 & 2010 & 2011 & 2010 & 2011 & & 10 & 2011 & 2010 & 2011 \\
\hline Americabetis alphus & 0,0 & 0,0 & 0,0 & 0,2 & 0,0 & 0,0 & 0 & 1,0 & 0,0 & 0,0 & 0,9 \\
\hline Andesiops peruvianus & 43,4 & 49,3 & 8,7 & 2,2 & 2,6 & 0,1 & 0 &, 7 & 1,6 & 1,8 & 0,8 \\
\hline Baetodes spp. & 1,2 & 9,4 & 25,1 & 74,7 & 67,8 & 63,5 & 64 & 4,0 & 56,6 & 8,6 & 45,5 \\
\hline Camelobaetidus. penai & 2,1 & 1,5 & 34,2 & 7,4 & 8,5 & 0,4 & 14 & 4,4 & 0,1 & 0,1 & 0,0 \\
\hline Nanomis. galera & 0,0 & 0,0 & 4,3 & 0,5 & 1,4 & 0,0 & 0 & ,6 & 0,0 & 0,2 & 0,6 \\
\hline Merdialaris tintinnabula & 0,9 & 1,6 & 0,1 & 0,3 & 0,0 & 0,0 & 0 &, 0 & 0,0 & 0,0 & 0,0 \\
\hline Thraulodes cochunaensis & 0,0 & 0,0 & 0,0 & 0,0 & 0,0 & 0,0 & 0 &, 0 & 0,0 & 0,2 & 0,0 \\
\hline Thraulodes consortis & 0,0 & 0,0 & 0,0 & 0,7 & 0,0 & 0,0 & 0 & 1,0 & 0,0 & 0,0 & 0,0 \\
\hline Thraulodes liminaris & 0,0 & 0,0 & 0,0 & 0,0 & 0,0 & 0,0 & 0 & 1,0 & 0,0 & 0,0 & 0,0 \\
\hline Haplohyphes. baritu & 0,0 & 0,0 & 0,0 & 0,0 & 0,0 & 0,0 & 0 & 1 & 0,0 & 0,0 & 0,0 \\
\hline Leptohyphes eximius & 0,0 & 0,0 & 0,2 & 1,7 & 0,2 & 22,0 & 6 & 2 & 14,3 & 7,0 & 0,0 \\
\hline \multirow[t]{2}{*}{ Caenis ludrica } & 0,0 & 0,0 & 0,0 & 0,0 & 0,0 & 0,0 & 0 & 1,0 & 0,0 & 0,6 & 0,0 \\
\hline & \multicolumn{2}{|c|}{$Q$} & \multicolumn{2}{|c|}{$\mathrm{L}$} & \multicolumn{2}{|c|}{$\mathrm{G} 1$} & \multicolumn{3}{|c|}{ G2 } & \multicolumn{2}{|c|}{$\mathrm{Ch}$} \\
\hline PRIMAVERA & 2010 & 2011 & 2010 & 2011 & 2010 & 2011 & 2010 & 2011 & & 2010 & 2011 \\
\hline Americabetis alphus & 0,0 & 0,2 & 0,0 & 0,0 & 0,2 & 0,1 & 0,0 & 0,0 & & 0,0 & 0,0 \\
\hline Andesiops peruvianus & 4,3 & 0,8 & 3,9 & 0,3 & 0,8 & 1,1 & 5,2 & 2,1 & & 10,7 & 2,7 \\
\hline Baetodes spp. & 66,6 & 59,0 & 60,7 & 72,4 & 67,9 & 79,7 & 1,0 & 1,2 & & 4,5 & 14,8 \\
\hline Camelobaetidius penai & 2,0 & 8,1 & 1,4 & 3,4 & 1,6 & 3,2 & 0,0 & 0,0 & & 0,4 & 0,7 \\
\hline Nanomis galera & 0,5 & 0,7 & 0,6 & 0,0 & 0,7 & 0,2 & 3,7 & 0,1 & & 3,3 & 1,3 \\
\hline Meridialaris. tintinnabula & 0,0 & 0,3 & 0,0 & 0,0 & 0,0 & 0,4 & 0,0 & 0,0 & & 0,0 & 0,0 \\
\hline Thraulodes cochunaensis & 0,0 & 1,0 & 0,0 & 0,5 & 1,8 & 0,7 & 1,9 & 1,4 & & 2,3 & 1,3 \\
\hline Thraulodes consortis & 1,7 & 1,1 & 0,7 & 0,0 & 0,2 & 0,3 & 1,2 & 0,9 & & 0,0 & 2,7 \\
\hline Thraulodes liminaris & 0,1 & 0,0 & 0,2 & 0,0 & 0,3 & 0,0 & 0,6 & 0,0 & & 0,4 & 0,0 \\
\hline Haplohyphes baritu & 0,0 & 0,0 & 0,0 & 0,0 & 0,0 & 0,0 & 0,0 & 0,0 & & 0,0 & 0,0 \\
\hline Leptohyphes. eximius & 1,2 & 0,0 & 9,1 & 9,9 & 2,4 & 3,9 & 3,3 & 0,9 & & 9,4 & 12,6 \\
\hline Caenis ludrica & 0,0 & 0,0 & 0,0 & 0,0 & 0,0 & 0,0 & 0,0 & 0,0 & & 0,0 & 0,0 \\
\hline
\end{tabular}

m s.n.m. y se cita a B. copiosus por primera vez para la provincia de Jujuy. Las restantes especies de la familia presentaron diferentes patrones de distribución, representatividad y rangos de tolerancia. Se cita a A. alphus por primera vez para la provincia de Jujuy, con bajas densidades, evidenciando preferencia por altitudes bajas, aguas oxigenadas y baja conductividad. Andesiops peruvianus se registró en todo el perfil altitudinal estudiado y en ambas estaciones, evidenciando tolerancia a incrementos en la conductividad del agua. Las variaciones en la densidad mostraron una preferencia por altitudes superiores a los 1700 m s.n.m. Camelobaetidius penai y $N$. galera registraron una variación estacional respecto a la distribución altitudinal. Durante los otoños mostraron preferencias por altitudes bajas mientras que durante las primaveras se amplía la distribución a sitios de mayor altura (Tablas 2 y 4). Ambos Leptohyphidae registraron una mayor representatividad durante las primaveras, indicando una marcada preferencia por temperaturas más altas. Mientras que $L$. eximius presentó una restringida distribución altitudinal, $H$. baritu registró, con menor densidad, preferencia por altitudes bajas (Tabla 4 y 5). Todas las especies registradas de la familia Leptophlebiidae: M. tintinnabula, T. cochunaensis, $T$. consortis y $T$. liminaris; estuvieron mejor representadas en la subcuenca Guerrero, evidenciando preferencia por las condiciones 
Tabla 4. Rango de parámetros físicoquímicos para las especies de Ephemeroptera registrados en la subcuenca del río Reyes, Jujuy, Argentina.

Table 4. Range of physicochemical parameters for the Ephemeroptera species recorded in the Reyes subbasin, Jujuy, Argentina.

\begin{tabular}{lcccc}
\hline & $\mathrm{pH}$ & $\begin{array}{c}\text { Temperatura } \\
\text { del agua }{ }^{\circ} \mathrm{C}\end{array}$ & $\begin{array}{c}\text { Oxígeno } \\
\text { Disuelto } \\
\mathrm{mg} / \mathrm{L}\end{array}$ & $\begin{array}{c}\text { Conductividad } \\
\mu \mathrm{S} / \mathrm{cm}\end{array}$ \\
\hline Americaabetis alphus & $6,0-9,0$ & $8,0-18,0$ & $3,3-8,5$ & $120-225$ \\
Andesiops peruvianus & $6,0-8,5$ & $8,0-25,0$ & $3,3-9,4$ & $117-283$ \\
Baetodes spp. & $6,0-9,0$ & $8,0-26,0$ & $3,3-8,5$ & $117-275$ \\
Camelobaetidius penai & $6,0-9,0$ & $8,0-25,0$ & $3,3-9,4$ & $117-283$ \\
Nanomis galera & $6,0-8,7$ & $8,0-25,0$ & $3,3-8,0$ & $117-275$ \\
Meridialaris & $6,0-9,0$ & $8,0-18,0$ & $3,3-8,3$ & $117-283$ \\
tintinnabula & & & & \\
Thraulodes & $6,5-8,7$ & $8,0-26,0$ & $4,0-9,4$ & $117-283$ \\
cochunaensis & & & & $120-150$ \\
Thraulodes. consortis & $6,0-9,0$ & $8,0-26,1$ & $3,3-8,0$ & $117-150$ \\
Thraulodes liminaris & $6,5-9,0$ & $8,0-26,2$ & $3,3-7,0$ & $117-283$ \\
Haplohyphes baritu & $7,5-8,0$ & $14,0-15,0$ & $7,0-7,5$ & $117-283$ \\
Leptohyphes eximius & $6,0-9,0$ & $8,0-26,0$ & $3,3-7,0$ & $120-250$ \\
Caenis. ludicra & $7,0-8,0$ & $20,0-25,0$ & $7,0-7,5$ & 150 \\
\hline
\end{tabular}

ambientales presentes en la subcuenca y menor torrencialidad.

Sin embargo, las especies de esta familia evidenciaron cambios estacionales respecto a la densidad. Mientras que M. tintinnabula mostró preferencias por altitudes medias y las condiciones imperantes durante los otoños y época lluviosa, $T$. cochunaensis y $T$. consortis presentaron mejor representatividad durante las primaveras. De las cuatro especies,
T. consortis fue la que registró a mayores altitudes. De la familia Caenidae se registró una sola especie, C. ludicra, con una distribución restringida y baja representatividad en la subcuenca con menor torrencialidad (Guerrero) y en sitios poco profundos y cercanos a las riberas con predominio de vegetación semisumergida y hojas (Tabla 5 ).

El estudio de la variabilidad espacio-temporal de los parámetros físico químicos mediante

Tabla 5. Densidad de individuos por especie (ind $/ \mathrm{m}^{2}$ ) en función de la altitud, temperatura promedio del agua $\left(\mathrm{T}-{ }^{\circ} \mathrm{C}\right)$ y estación del año (E): otoño (O); primavera (P). Americaabetis alphus: A.a; Andesiops peruvianus: A.p:; Baetodes spp: B. spp; Camelobaetidius penai: C.p; Nanomis galera: N.g; Meridialaris tintinnabula: M.t; Thraulodes cochunaensis: T.c; Thraulodes. consortis T.co; Thraulodes liminaris: T.l; Haplohyphes baritu: H.b; Leptohyphes eximius: L.e; Caenis lúdicra: C.I.

Table 5. Density of individuals by species (ind $/ \mathrm{m}^{2}$ ) according to altitude, average water temperature $\left({ }^{\circ} \mathrm{C}\right)$ and season of the year (O: autumn-P: spring).

\begin{tabular}{|c|c|c|c|c|c|c|c|c|c|c|c|c|c|c|}
\hline Altitud & $E$ & $\mathrm{~T}-{ }^{\circ} \mathrm{C}$ & A. $a$ & A.p & B. spp. & C.p & N.g & M.t & T.C & T.cc & T.I & H.b & L.e & C.I \\
\hline $1800-$ & 0 & 11,1 & 0 & 157,4 & 88 & 11,1 & 0 & 12,1 & 3,7 & 0 & 0 & 0 & 0 & 0 \\
\hline 2200 & $P$ & 14 & 0 & 1271,3 & 187,1 & 44,45 & 0 & 38 & 0 & 0 & 0 & 0 & 0 & 0 \\
\hline \multirow{2}{*}{1700} & 0 & 11,1 & 0 & 1463 & 8668,6 & 216,7 & 0 & 32,1 & 9,3 & 0 & 0 & 0 & 0 & 0 \\
\hline & $P$ & 15,5 & 0 & 440,8 & 3783,4 & 1687 & 238 & 14,8 & 0 & 27,8 & 0 & 0 & 43,5 & 0 \\
\hline \multirow{2}{*}{1600} & 0 & 12 & 6 & 213,3 & 4401,5 & 563,6 & 42,6 & 121,3 & 2,5 & 3,4 & 1,9 & 0 & 4,9 & 0 \\
\hline & $P$ & 16,3 & 1,9 & 272,2 & 8591,4 & 444,5 & 85,2 & 4,3 & 23,5 & 29,7 & 5,6 & 0 & 317,3 & 1,9 \\
\hline \multirow{2}{*}{1500} & 0 & 12,3 & 2,5 & 112,4 & 6782,7 & 772,5 & 5,3 & 448,5 & 3,1 & 1,9 & 0 & 0 & 28,1 & 0 \\
\hline & $P$ & 20,9 & 2,5 & 81,5 & 3066,6 & 112,4 & 25,3 & 5,5 & 43,2 & 19,1 & 5,6 & 0 & 236,4 & 0,6 \\
\hline \multirow{2}{*}{1400} & 0 & 11,5 & 1,9 & 104,7 & 6750,9 & 135,2 & 19,4 & 38,9 & 1 & 4,6 & 0 & 0 & 19,5 & 0 \\
\hline & $P$ & 18,4 & 0 & 124,1 & 4165,7 & 241,7 & 32,4 & 0 & 6,5 & 0 & 0 & 1,9 & 911,1 & 0 \\
\hline
\end{tabular}




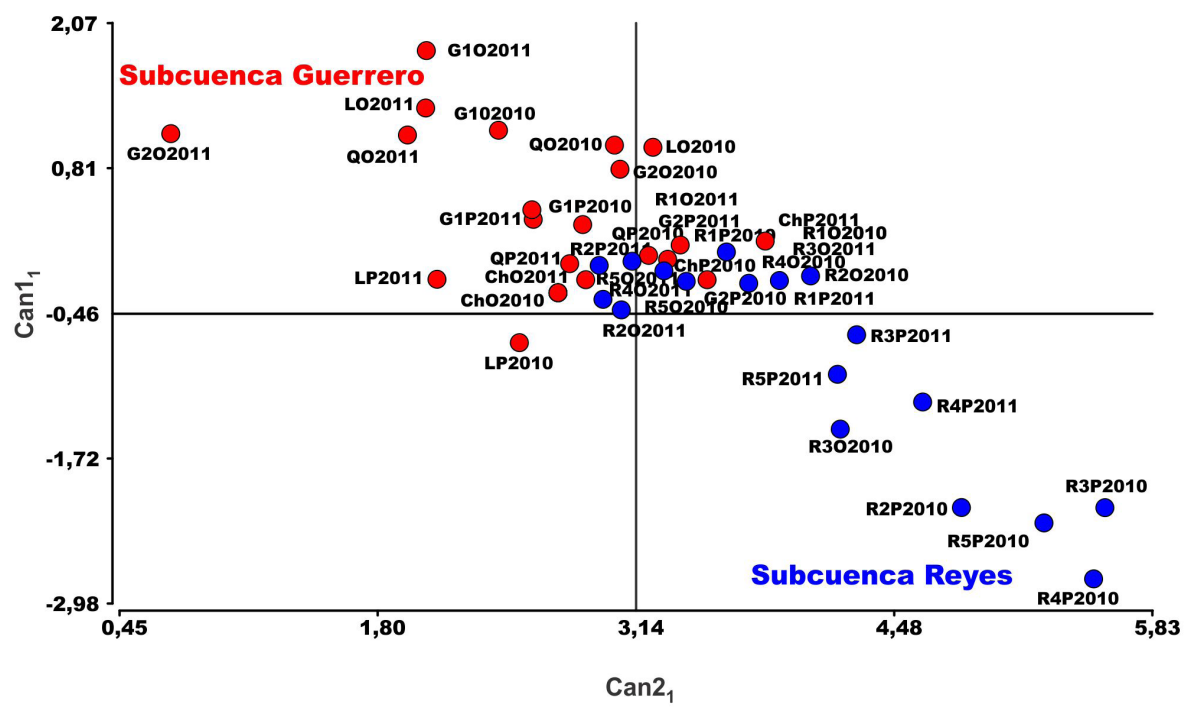

Figura 2. Análisis de la variabilidad espacio-temporal de los parámetros físicoquímicos del agua. Análisis de Componentes Principales. Referencias: $\mathrm{R}=$ río Reyes; $\mathrm{G}=$ río Guerrero; Q= río Quesera; L=río Lampazar; Ch= río Chinchuli; $\mathrm{O}=$ otoño; $\mathrm{P}=$ primavera; 2010-2011 = año campaña.

Figure 2. Analysis of the variability spatio-temporal of the physico-chemical parameters of water. Principal component analysis. References: R= Reyes River; G= Guerrero River; Q= Quesera River; L= Lampazar River; Ch= Chinchuli River; $\mathrm{O}=$ fall; $\mathrm{P}=$ spring; 2010-2011= year monitoring.

componentes principales permitió explicar el $68 \%$ de la variabilidad total. El análisis puso de manifiesto la variabilidad espacial entre sitios y una marcada estacionalidad en la cuenca del Reyes. Las primaveras estuvieron caracterizadas por mayores registros de conductividad y de oxígeno disuelto y de temperatura del agua (Figura 2). La conductividad y concentración de oxígeno disuelto resultaron las variables con mayor peso estadístico para explicar la variabilidad entre sitios de la cuenca Reyes $\left(R^{2}=0,62\right)$. El rango de valores registrados de $\mathrm{pH}$ y oxígeno disuelto se encuentran dentro de los niveles guía sugeridos por la Ley de Medio Ambiente de la provincia de Jujuy, 5063/73, para la protección de la vida acuática en agua dulce superficial.

Se evidenció una alta correlación lineal $\left(\mathrm{R}^{2}=0,72\right.$; $\mathrm{p}=0,01)$ entre las variables físicoquímicas consideradas $(\mathrm{pH}$, conductividad y oxígeno disuelto) y las abundancias absolutas de las especies de Ephemeroptera, lo que permite destacar el rol del orden como buenos indicadores de cambios en las características del agua (Figura 3).

\section{DISCUSIÓN}

La elevada representatividad del Orden Ephemeroptera registrada para la cuenca del Reyes y valores máximos de abundancia y densidad de especies durante la estación seca, coinciden con lo propuesto por Jacobsen y Encalada (1998), Mesa et al. (2009) para ríos de montañas neotropicales con marcada estacionalidad de lluvias.

De las cuatro familias registradas para la cuenca del Reyes, Baetidae presentó la mayor riqueza de géneros y representatividad. Esto pone de manifiesto la capacidad que presentan sus ninfas para adaptarse a distintos tipos de hábitats (Alba-Tercedor, 2004; Hamada et al., 2018). Esta amplia tolerancia de la familia registrada, permite coincidir con la asignación de un puntaje bajo en la construcción de un índice biótico adaptado a la región de las Yungas argentinas (Domínguez y Fernández, 1998). De los veintiún géneros endémicos de Baetidae citados para Sudamérica (Hamada et al., 2018), cinco se registraron en la cuenca en 


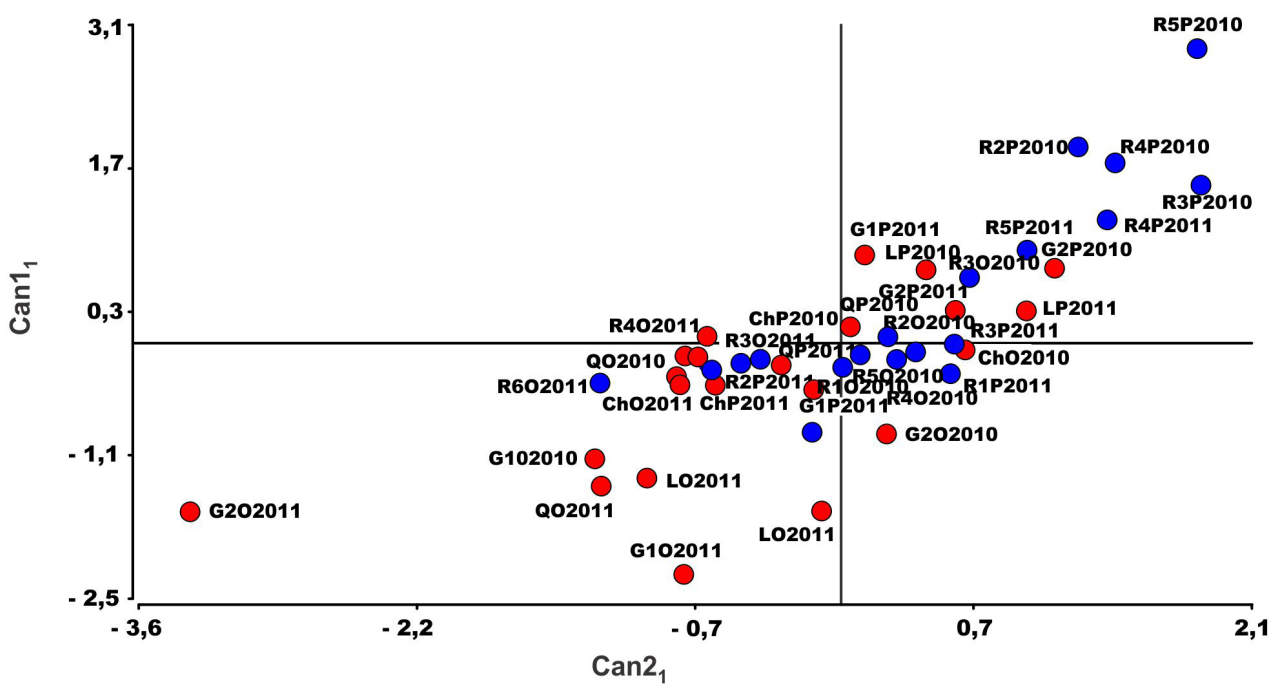

Figura 3. Análisis de correlación canónica de la distribución espacio temporal de las especies de Ephemeroptera. Ordenamiento de los sitios: el agrupamiento en rojo pertenece a la subcuenca Guerrero y el azul a la subcuenca Reyes.

Figure 3. Canonical correlation analysis of the temporal space distribution of the Ephemerotera species. Assortment of the sites: the red grouping belongs to the Guerrero sub-basin and the blue to the Reyes subbasin.

estudio, con ocho especies. Baetodes fue el género mejor representado y más diverso en la cuenca, evidenciando una amplia tolerancia a condiciones físico químicas del agua y preferencia por flujos de corrientes rápidas y oxigenadas. Los mayores registros de densidad del género coinciden con la época lluviosa. Estas preferencias de hábitat registradas y puestas de manifiesto en el presente estudio coinciden con las mencionadas por Zúñiga de Cardoso et al. (1997), Jacobsen (2005), Pérez y Segnini (2007) y Ríos Touma et al. (2011) en ríos neotropicales de montaña de Ecuador y Colombia. El bajo puntaje a la familia Baetidae asignado en el índice biótico BMWP, el más utilizado para las Yungas hasta los 2000 m (Domínguez y Fernández, 1998), también genera controversias con los resultados obtenidos en el presente estudio. En la cuenca analizada, las otras especies de la familia, registradas con menor representatividad, evidenciaron diferentes patrones de distribución, representatividad y rangos de tolerancia. A partir de lo cual, el valor indicador asignado a la familia refleja la tolerancia del género más representativo, no considerando la heterogeneidad adaptativa observada en las especies registradas con menor representatividad. Por ejemplo, A. peruvianus, C. penai y N. galera fueron registrados a lo largo del perfil longitudinal de los ríos con elevada representatividad, sin embargo el análisis de la variabilidad en las densidades pone de manifiesto diferencias respecto a preferencias en altitud, rango de conductividad y estacionalidad. Mientras que $A$. peruvianus manifiesta preferencia por hábitats de cabeceras de ríos, donde los procesos erosivos son importantes y aportan iones disueltos, con temperaturas bajas (Domínguez et al., 2006), N. galera prefiere en la cuenca Reyes, menores altitudes y condiciones presentes en sitios con características de drenaje bajo. Para A. alphus, si bien la baja densidad registrada por sí sola la transforma en una especie con pobre valor como indicador, la restringida distribución espacio-temporal manifiesta un estrecho rango de tolerancia a los cambios en las condiciones del agua y sitios. 
Las tres especies de Leptohyphiidae registradas en el estudio evidenciaron preferencia por las condiciones presentes en los sitios de baja altitud. Haplohyphes baritu y L. eximius registraron la mayor densidad de individuos durante las primaveras, mientras que $M$. tintinnabula presentó en la cuenca preferencias por las bajas temperaturas siendo este comportamiento también observado en otros ríos de yungas de Tucumán (Domínguez y Ballesteros Valdez, 1992)

El género Thraulodes está representado en la cuenca Reyes por tres de las cinco especies citadas para Argentina. Es uno de los géneros más diversos y ampliamente distribuidos en la región Neotropical (Domínguez, 1987), citado como sensible a la contaminación y perturbaciones de origen antrópico (Domínguez y Fernández, 1998; Zuñiga et al., 1997). En sitios de la cuenca Reyes T. cochunaensis y T. consortis fueron registradas con similares características, asociando su presencia a condiciones de aguas limpias y oxigenadas.

Caenis ludicra fue la única especie registrada de la familia Caenidae. De acuerdo a algunos autores se trata de una especie ampliamente distribuida en el NOA (Molineri \& Malzacher, 2007); lo que estaría indicando una amplia capacidad de adaptación a las características de los ríos de montaña del NOA. Sin embargo, para el área en estudio, la especie presentó una distribución restringida a una sola de las subcuencas y durante las primaveras. Las preferencias de hábitat de la especie coinciden con las citadas por Hamada et al. (2018) respecto a las características de los sitios donde fue registrada - presencia de hojas y vegetación semisumergida- de la subcuenca. Es importante mencionar que la especie no se registró en la subcuenca Reyes, caracterizada por su mayor torrencialidad y amplia playa de inundación desprovista de vegetación. El comportamiento estacional y distribución restringida pone de manifiesto que la especie posee un estrecho rango de tolerancia a condiciones ambientales y físicoquímicas del agua. De ello se puede deducir que la calificación baja de la familia propuesta por el BMWP modificado por el NOA (Domínguez y Fernández, 1998) igual que los baétidos, no refleja el verdadero rol indicador de la familia Caenidae para esta cuenca de
Yungas de Jujuy.

El análisis de componentes principales realizado puso de manifiesto diferencias entre las subcuencas y una marcada estacionalidad en la cuenca del Reyes. La estacionalidad es una característica observada en otras cuencas de montaña de Yungas argentinas y neotropicales de Colombia y Ecuador (Casas et al., 2006; Domínguez y Fernández, 1998; Domínguez et al., 2006; Roldán, 1980; Romero et al., 2011; Muñoz y Ospina, 1999; Ríos Touma et al., 2011; Zúñiga et al., 2013). La heterogeneidad espacio-temporal encontrada en la cuenca permite considerar la influencia de ésta en la ecología de los ríos (Pringle et al., 1988). La temperatura del agua es un componente de suma importancia que regula la solubilidad del oxígeno, dióxido de carbono, conductividad, disolución de minerales (Butturini et al., 2009; Moraña, 1998) y biología de las comunidades de Ephemeroptera (Salles et al., 2018). En la cuenca Reyes los mayores registros de temperatura, oxígeno disuelto y conductividad están asociados a las primaveras que, al coincidir con el estiaje, el aumento de la evapotranspiración provoca una mayor concentración de iones proveniente de la actividad erosiva de los ríos de montaña. Los registros de $\mathrm{pH}$, conductividad, temperatura del agua y oxígeno disuelto obtenidos en la cuenca del Reyes, coinciden con los obtenidos para los ríos Churqui y Carreras de las Yungas de Tucumán (Isasmendi et al., 2007).

\section{CONCLUSIONES}

Se confirmó para la cuenca del Reyes, el rol de los Ephemeroptera como indicadores en virtud a su elevada representatividad y a la capacidad de las especies para describir la variabilidad espaciotemporal de los sitios según las características físicoquímicas del agua. El conjunto de variables registradas $(\mathrm{pH}$, temperatura del agua, oxígeno disuelto y conductividad) se correlacionan con la variabilidad de las abundancias de las especies de efemerópteros.

\section{AGRADECIMIENTOS}

Los autores agradecen a los Dres. Carlos Molineri 
y Eduardo Domínguez su asesoramiento para la identificación y confirmación de las especies de efemerópteros. A la Agencia Nacional de Promoción Científica y Técnica (ANPCyT, Programa RRHH-PFDT red 101) y a la Secretaría de Ciencia y Técnica y Estudios Regionales de la Universidad Nacional de Jujuy (Resolución C.S. n 0269-15; Resolución R.N. 461-13, Proyecto SeCTER A/105; B/039).

\section{REFERENCIAS}

Alba-Tercedor, J., Jáimez-Cuellar, P., Álvarez, M., Avilés, J., Bonada, N., Casas, J. y Rieradevall, M. (2004). Caracterización del estado ecológico de ríos mediterráneos ibéricos mediante el índice IBMWP (antes BMWP'). Limnetica, 21, 175-185.

Braun Wilke, R. (2001). Carta de aptitud ambiental de la Provincia de Jujuy. Departamento de suelos y ecología. Facultad de Ciencias Agrarias. UNJu.

Butturini, A., Sabater, S. y Romaní, A. (2009). La química de las aguas. Los nutrientes. En: Elosegui, A. y Sabater, S. (Eds.). Conceptos y Técnicas de Ecología fluvial. España: Fundación BBVA.

Cabrera, A. (1976). Regiones fitogeográficas de la República Argentina. Enciclopedia de la Agricultura, Jardinería y Fruticultura 2: 1-85.

Casas, L. K. E., Córdoba, S., Asprilla S. y Mosquera Murillo, Z. (2006). Composición y distribución del orden Ephemeroptera en los ríos Tutunendó y Catugadó, QuibdóChocó (Colombia). Asociación Colombiana de Limnología, 1, 92-97.

Cruz, P. V., Falcao Salles, F. \& Hamada, N. 2014. Callibaetis Eaton (Ephemeroptera: Baetidae) from Brazil. Journal of Natural History, 48(11-12), 591-660.

Di Rienzo J. A., Casanoves, F., Balzarini, M. G., González, L., Tablada, M. y Robledo, C. W. (2008). InfoStat, versión 2008, Grupo InfoStat, FCA, Universidad Nacional de Córdoba, Argentina.
Domínguez, E. (1984). Dos especies nuevas del género Haplohyphes Allen (Ephemeroptera: Trycorythidae) de la Argentina. Revista de la Sociedad Entomológica Argentina, 43(1-4), 103-112.

Domínguez, E. (1987). El género Thraulodes (Ephemeroptera: Leptophlebiidae) en la República Argentina. Acta Zoológica Lilloana, 39(1), 47-65.

Domínguez, E. \& Ballesteros Valdez, J. M. (1992). Altitudinal replacement of Ephemeroptera in a subtropical river. Hydrobiologia, 246(1), 83-88.

Domínguez, E. y Fernández, H. R. (1998). Calidad de los ríos de la Cuenca del Salí (Tucumán, Argentina) medida por un índice biótico. Serie Conservación de la Naturaleza 12. Fundación Miguel Lillo, Tucumán, Argentina.

Domínguez, E. y Fernández, H. R. (2009). Macroinvertebrados bentónicos sudamericanos. Sistemática y biología. Fundación Miguel Lillo, San Miguel de Tucumán, Tucumán, Argentina.

Domínguez, E., Molineri, C., Pescador, M. L, Hubbard, M. D. \& Nieto, C. (2006). Ephemeroptera of South America. In: Adis, J., Arias, J. R., Rueda-Delgado, G. \& Wantzen, K.M. (Eds.). Aquatic Biodiversity of Latin America. Pensoft Press, Sofia and Moscow. ABLA Series.

Dos Santos, D. A., Molineri, C., Reynaga, M. C. \& Basualdo, C. (2011). Which index is the best to assess stream health? Ecological Indicators, 11(2), 582-589.

Jacobsen, D. (2005). Temporally variable macroinvertebrate- stone relationships in streams. Hydrobiologia, 544 (1), 201-214.

Jacobsen, D. \& A. Encalada, A. (1998). The macroinvertebrate fauna of Ecuadorian highland streams in the wet and dry season. Archiv fr Hydrobiologie, (142), 53-70. 
Hamada, N., Thorp, J. H. \& C. Rogers (Eds.). (2018). Keys of Neotropical Hexapoda. Thorp and Covich's Freshwater Invertebrates, Volume 3. Academic Press.

Isasmendi, S. C.; Tracanna, B. C.;Vendramini, F. H.; Navarro, M. G.; Barrionuevo, M. A. y Meoni, G.S. (2007). Caracterización física y química de ríos de montaña (Tafí del Valle-Tucumán-Argentina). Limnetica, 26(1), 129-142.

Mesa, L. M. (2011). Diversidad de macroinvertebrados bentónicos en la cuenca del río Lules. En: Fernández, H. R. y Barber, H. M. (Eds.). La Cuenca del río Lules. Una aproximación multidisciplinaria a su complejidad (pp. 137-148). San Miguel de Tucumán, Argentina: EDUNT.

Mesa, L. M.; Fernández, H. R. \& Manzo, M. V. (2009). Seasonal patterns of benthic arthropods in a subtropical Andean basin. Limnologica, 39, 152-162.

Molineri, C. (2010). Las especies de Leptohyphidae (Ephemeroptera) de las yungas de Argentina y Bolivia: diagnosis, distribución y claves. Revista de la Sociedad Entomológica Argentina, 69(3-4), 233-252.

Molineri, C. \& Malzacher, P. (2007). South American Caenis Stephens (Ephemeroptera, (aenidae), new species and stage descriptions. Zootaxa, 1660, 1-31.

Moraña, L. B., Salusso, M. M. y García de Emiliani, M. O. (2000). Evaluación de la calidad del agua en la cuenca del río Grande (Alta Cuenca del Bermejo, Jujuy) Argentina. En: Farías, H. D., Pilan, M. T., Borsellino, M. J., Pece, F. J., Storniolo, A. R. y Mattar, M. T. (Eds.).Uso y preservación de los recursos hídricos en los umbrales del siglo XXI (pp. 285286). Santiago del Estero, Argentina: Instituto de Recursos Hídricos, Facultad de Ciencias Exactas y Tecnologías, Universidad Nacional de Santiago del Estero.
Muñoz, D. y Ospina, R. (1999). Guía para la identificación genérica de los Ephemeroptera de la Sabana de Bogotá, Colombia. Ninfas y algunos géneros de adultos. Actualidades Biológicas, 21(70), 47-60.

Nieto, C. (2003). El género Camelobaetidius (Ephemeroptera: Baetidae) en la Argentina. Acta Zoológica Mexicana, 88, 233-255.

Nieto, C. (2004). The genus Baetodes (Ephemeroptera: Baetidae) in South America with the description of new species from Argentina, Bolivia and Perú. Studies on Neotropical Fauna and Environment, 39, 63-79.

Pérez, B. y Segnini, S. (2007). Variación espacial de la composición y diversidad de géneros de Ephemeroptera (Insecta) en un río tropical altoandino. Entomotropica, 20(1), 49-57.

Pescador, M. L. \& Peters, M. L. (1986). Revision of the genera Meridialaris and Massartellopsis (Ephemeroptera: Leptophlebiidae: Atalophlebiinae) from South America. Transactions of the American Entomological Society, 112, 147-189.

Pringle, C. M., Naiman R. J., Bretschko, G., Karr, J. R., Oswood, M. W., Webster, J. R., Welcomme R. L. \& Winterbourn, M. J. (1988). Patch dynamics in lotic streams: The stream as a mosaic. Journal of North American. Benthological Society, (7), 503-524.

Ríos Touma, B., Encalada, A. C. \& Prat, N. (2011). Macroinvertebrate assemblages of an Andean high-altitude tropical stream: The importance of season and flow. International Review of Hydrobiology, 96(6), 667-685.

Roldán, G. (1980). Estudios limnológicos de cuatro ecosistemas neotropicales diferentes con especial referencia a su fauna de efemerópteros. Actualidades Biológicas, 9(34): 103-117. 
Romero, V. F., Fernández, H. R., Manzo, V., Molineri, C., Correa, M. \& Nieto, M. C. (2011). Estudio integral de la cuenca del Río Lules (Tucumán): aspectos biológicos. En: Fernández, H. R. y Barber H., M. (Eds.). La Cuenca del río Lules: una aproximación multidisciplinaria a su complejidad (pp. 111135). San Miguel de Tucumán, Tucumán, Argentina: EDUNT.

Salles, F. F., Domínguez, E., Molineri, C., Boldrini, R., Nieto, C. \& Dias, L. G. (2018). Order Ephemeroptera. In: Hamada, N., Thorp, J. H \& Rogers, D. C. (Eds.). Thorp and Covich's Freshwater Invertebrates. Vol. III. Keys to Neotropical Hexapoda (pp. 61-117). London: Academic Press.

Sartori, M. \& Brittain, J. E. (2015). Order Ephemeroptera. In: Thorp, J. \& Rodgers, D. C. (Eds.). Thorp and Covich's Freshwater Invertebrates: Ecology and General Biology 4th Edition (pp. 873-891). New York: Academic Press.
Studholme, A. M., León, L. F. H., Rivera A. C. R. \& Moreno, C. G. R. (2014). Altitudinal and temporal variability of four macroinvertebrate communities in Andean streams, Sangay National Park, Ecuador. Entomotropica, 29(3), 149-158.

Wallace, J. B. \& Webster, J. R. (1996). The role of macroinvertebrates in stream ecosystem function. Annual Review of Entomology, 41(1), 115-139.

Zúñiga de Cardozo, M. C., Rojas de Hernández, A. M., \& Mosquera, S. (1997). Biological aspects of Ephemeroptera in rivers of southwestern Colombia (South America). In: Landolt, P. \& Sartori, M. (Eds.). Ephemeroptera and Plecoptera: Biology-Ecology-Systematics (pp. 261-268). Fribourg, Switzerland: MauronTinguely and Lachat S.A.

Zúñiga, M. D. C., Chará, J., Giraldo, L. P., Chará-Serna, A. M. \& Pedraza, G. X. (2013). Composición de la comunidad de macroinvertebrados acuáticos en pequeñas quebradas de la región andina colombiana, con énfasis en la entomofauna. Dugesiana, 20(2), 263-277. 